\title{
Mapping and genetic overlapping of QTLs related to drought and heat tolerances in spring wheat
}

\author{
Lei Shi ( $\nabla$ s5266@126.com) \\ National Institute of Crop Science \\ Haibo Bai \\ Ningxia Academy of Agriculture and Forestry Sciences \\ Yongxing Zhu \\ Ningxia Academy of Agriculture and Forestry Sciences

\section{Xuelian LV} \\ Ningxia Academy of Agriculture and Forestry Sciences \\ Shuhua Li \\ Ningxia Academy of Agriculture and Forestry Sciences \\ Jian Hui \\ Ningxia Academy of Agriculture and Forestry Sciences \\ Xingguo Ye \\ Chinese Academy of Agricultural Sciences \\ Jianli Dong \\ Ningxia Academy of Agriculture and Forestry Sciences
}

\section{Research Article}

Keywords: wheat, drought tolerance, heat tolerance, QTL mapping, genetic overlapping

Posted Date: March 4th, 2021

DOI: https://doi.org/10.21203/rs.3.rs-280254/v1

License: (9) (1) This work is licensed under a Creative Commons Attribution 4.0 International License. Read Full License 


\section{Abstract}

Quantitative trait locus (QTL) mapping associated with wheat drought and heat tolerances was characterized in this study. There were 25, 25, 23 and 17 QTLs controlling chlorophyll content, leaf water content, spike grain weight and thousand-kernel weight, respectively. The QTLs related to drought tolerance were located on 7 wheat chromosomes with a phenotypic contribution rate of $9.38-34.20 \%$, related to heat tolerance on 9 wheat chromosomes with a phenotypic contribution rate of $9.03-35.55 \%$, and related to combined drought and heat tolerances on 11 wheat chromosomes with a phenotypic contribution rate of $9.09-33.11 \%$, respectively. Among them, 35 QTLs were newly located, in which 16 were associated with heat tolerance, and 19 associated with combined drought and heat tolerances. We found 2 overlapping sites related to drought tolerance on chromosomes $2 \mathrm{~A}$ and $3 \mathrm{~B}, 3$ overlapping sites related to heat tolerance on chromosomes $2 A, 7 B$ and $6 \mathrm{D}$, and 4 overlapping sites related to combined tolerances for drought and heat on chromosomes 2A, 2B and 4D. The overlapping QTLs with stable expression under different environmental stresses were located on chromosomes 2A (gwm294-wmc644), 3B (wmc808-wmc78) and 7B (wmc83-wmc276). The overlapping loci involving in pleiotropism or a close linkage have high phenotypic contribution rates and environmental stability.

\section{Introduction}

Due to a shortage of water resources and increasing temperatures driven by climate change, many areas across the globe have experienced serious wheat yield losses in recent years, especially in arid regions (Hu et al. 2014; He et al. 2017). Wheat plants are very sensitive to heat stress, especially during the reproduction process and the initial stage of grain filling. With global warming, extremely high temperature events occur frequently, and the damage to the wheat is intensified when these temperature stresses occur during the wheat filling stage (Barlow et al. 2015; Ni et al. 2018). Thus, it is critical to research ways increase wheat's drought and heat tolerance.

Breeders and physiologists have conducted considerable research into the mechanism of wheat tolerance against abiotic stress and how to enhance it (Budak et al. 2015; Caverzan et al. 2016; Ni et al. 2018). The amount of transcription factors (TFs), genes, microRNAs (miRNAs), hormones, proteins, co-factors, ions and metabolites have been investigated for their roles in plant abiotic stress tolerance (Budak 2015; Caverzan et al. 2016; Zhang et al. 2016; Wan et al.2017; Zhang et al. 2019). Since drought and heat are two major detriments to wheat production worldwide, many studies have focused on improving wheat tolerance to these two stressors (Mwadzingeni et al. 2017; Tricker et al. 2018). A number of genes and TFs have been identified in wheat that respond to water and temperature stresses (Johnson et al. 2014; Liu et al. 2015; He et al. 2016; Szucs et al. 2010). However, there are still many bio-functional genetic loci that have been only primarily located on chromosomes but not precisely confirmed.

Quantitative trait loci (QTL) analysis is a good way to link phenotypic data and genotypic data to explain the genetic variation basis of complex traits in wheat plants (Miles et al. 2008). A host of wheat traits including botanic phenotype, yield and yield components have been shown to be associated with QTL sites under various biotic and abiotic stresses (Kumari et al. 2018; Liu et al. 2018; Sukumaran et al. 2018; Tricker et al. 2018; Hu et al. 2019; Liu et al. 2019; Muqaddasi et al. 2019; Ye et al. 2019). Among them, dozens of QTLs were located in the wheat genome under drought conditions (Quarrie et al. 2005; Dashti et al. 2007; Yang et al. 2007; Czyczyło-Mysza et al. 2011; Kadam et al. 2012; Mwadzingeni et al. 2016, 2017; Xu et al. 2017) and extreme heat conditions (Maccaferri et al. 2008; Mason et al. 2010; Shirdelmoghanloo et al. 2016; Ogbonnaya et al. 2017). Particularly, many wheat QTLs were identified under the combined conditions of drought and extreme heat, which were almost overlapping on each chromosome of wheat (Kirigwi et al. 2007; Pinto et al. 2010; Bennett et al. 2012; Merchuk-Ovnat et al. 2016; Tahmasebi et al. 2016; Liu et al. 2019). For instance, 25 QTLs for the wheat heat-tolerant trait during the grain filling period were located on chromosomes $1 \mathrm{~A}, 2 \mathrm{~A}$, 2B and 3B using a population of recombinant inbred lines (RILs), among which five QTLs controlling leaf length, width and visual wax content were stably expressed (Mason et al. 2010). Fourteen QTLs were identified to control wheat root length and root biomass under water stress conditions. Among these, five QTLs were also found to control maximum root length associated with drought tolerance. These overlapping QTLs were located on chromosomes 2D, 4B, 5D and 6B (Kadam et al. 2012). Based on wheat recombinant inbred lines (RILs) for chlorophyll content, canopy temperature difference, thousand-grain weight (TGW), and heat tolerance index of yield, a QTL map has previously been constructed for chromosomes 2B, 7B and 7D (Paliwal et al. 2012). In addition, three main heat-tolerant QTLs were detected in wheat based on a RILs population, which were distributed on $1 B, 5 B$, and 7B (Mohammadi et al. 2008). 
It has been shown that wheat chromosomes 2A, 3A, 3B, 6A, 6B and 7A are closely associated with the leaf stay-green QTL under heat stress (Vijayalakshmi et al. 2010). Using a set of wheat chromosomal substitution lines and a RILs population, chromosomes 3D, 4A, 5A and 5B were confirmed to be related to heat tolerance, and several QTLs that were closely associated with canopy temperature under heat stress were found to be located on chromosomes 3B and 7A (Pinto et al. 2010). Some QTLs controlling plant height under drought stress were detected on chromosomes 1B, 4B and 7D (Tahmasebi et al. 2017; Xu et al. 2017). Meanwhile, many QTLs controlling plant height associated with heat tolerance were located on all the wheat chromosomes except 1D, 4D, 5D and 6B, and most of them were also detected under combined conditions of water stress and high temperature (Maccaferri et al. 2008; Pinto et al. 2010; Ogbonnaya et al. 2017; Tahmasebi et al. 2017). Some QTLs controlling chlorophyll content associated with drought tolerance were located on chromosomes 1B, 2B, 5B, 7A and 7B (Ilyas et al. 2014; Tahmasebi et al. 2017; Xu et al. 2017). A set of QTLs controlling chlorophyll content were detected on chromosomes 1A, 1B, 1D, 2B, 3A, 3B, 4A, 4D, 5A, 5B, 6A, 6D, 7A, 7B and 7D under heat stress (Peleg et al. 2009; Pinto et al. 2010; Bennett et al. 2012; Tahmasebi et al. 2017).

A number of QTLs were detected under both stress conditions of drought and heat, and this genetic overlapping revealed that drought and heat conditions may trigger some conjoint pathways involving in wheat abiotic stress resistance (Tricker et al. 2018). Studies indicated that those wheat QTLs identified under drought and heat conditions were also associated with some agronomic and physiological traits or parameters such as grain yield, TGW, grain number, spike weight, biomass, plant height, days to heading and chlorophyll content (Tricker et al. 2018). Most of the aforementioned wheat QTLs were assigned to chromosomes by using wheat double-haploid (DH) populations and RIL populations. However, the mapping of the QTLs related to drought and heat tolerances was different in different studies because of the variations in wheat materials used, investigated traits, and adopted stress patterns and environments (Tahmasebi et al. 2017; Tricker et al. 2018).

Studies have shown that high temperature stress has the effect of increasing water stress, which will further aggravate the damage caused by high temperatures. The damage caused by high temperatures accompanied by drought is far greater than that caused by high temperature or drought alone (Pinto et al. 2015). Because synergistic or antagonistic effects usually occur under compound stress, the effects of compound stress cannot be predicted based on the response to a single stress (Grigorova et al. 2011). Our previous study found that the combined drought and high temperature stress caused a nearly three-fold reduction in thousand-grain weight compared to only a single stressor (Dong et al. 2008). Research on wheat QTLs has revealed that there is a large amount of genetic overlapping among relevant trait loci and among resistance loci. For example, there were different degrees of genetic overlapping among yield and yield components (Liu et al. 2011), and among biological resistance and non-biological resistance.

Polyploidy of common wheat is an important cause for genetic overlapping between tolerance and agronomic traits. Investigations on wheat QTL mapping under double stresses of drought and salt, salt and low temperature, and disease and drought have revealed a large degree of genetic overlapping between the QTL loci of stress-related traits (Xu et al. 2012). Locating genetic overlapping sites of wheat QTLs for drought and heat tolerances can provide useful information for improving the genetics of this crop through breeding, and in-depth research is needed (Lawas et al. 2018). This study was conducted to locate favorable QTL alleles associated with drought and heat tolerances, and to identify genetic overlapping between the two stresses on wheat chromosomes. The research was conducted using a RILs population derived from a cross of two spring wheat varieties (one drought-tolerant variety one heat-tolerant variety) in different environments and stress conditions in multiple years. The results reported here provide a solid basis for genetic improvement of wheat drought and heat tolerances by using molecular markerassisted selection (MAS) and gene pyramiding strategies.

\section{Materials And Methods}

\section{Plant materials}

A strain of Ningchun4, which is a heat-tolerant spring wheat variety with high yield and quality and wide adaptability, is maintained by our research team. This variety was developed in 1981, and it accounts for $60 \%$ of the total area of wheat in the Ningxia irrigation area. It has been used as the control variety in regional trials in the Ningxia irrigation wheat area and northern spring wheat area of China for more than 30 years. Ningchun27 was bred and provided by Guyuan Agricultural Science Institute, Ningxia. Ningchun27 is a drought-tolerant spring wheat variety with high yield and quality, wide adaptability, good resistance to stripe rust, and moderate resistance to powdery mildew. It is the main variety currently planted in the rain-fed area in southern Ningxia. 
Ningchun4 was used as the parent to hybridize with Ningchun27. The $F_{1}$ generation was self-crossed, and an $F_{10}$ generation with full genetic stability was obtained from the $F_{2}$ generation by the one-seed inheritance strategy. Then, a RILs population was constructed through genetic stability and leaf blight identification, and 128 RILs were reserved and used in this study.

\section{Experimental set-up}

During the years from 2015 to 2017, field trials were conducted at the Crop Research Institute, Ningxia Academy of Agricultural and Forestry Sciences, Yinchuan, Ningxia (E1); the Crop Research Institute, Academy of Agricultural and Pastoral Sciences, Hohhot, Inner Mongolia (E2); and the Crop Research Institute, Xinjiang Academy of Agricultural Sciences, Urumqi, Xinjiang (E3). Different sites are considered to be environmental factors and each site is set up with 4 stress treatments. Each material was planted in two rows by $1.0 \mathrm{~m}$ in length and $0.2 \mathrm{~m}$ in width, with 80 seeds sown in each row. This planting design was replicated three times at each environmental site. The spacing between replicate treatments was $6.0 \mathrm{~m}$. Fertilization of wheat, and weed and disease control at each site was consistent with local field management.

\section{Stress treatment design}

For drought stress treatment only, an irrigation volume of $900 \mathrm{~m}^{3} \mathrm{hm}^{-2}$ was applied at the jointing and heading stages, respectively. Drought stress lasted from seedling to maturity stages. The moisture content in the $0 \sim 50.0 \mathrm{~cm}$ soil layer was controlled at $7 \% \pm 1 \%$. An awning frame was erected for covering the experimental field with plastic and applied when rain was imminent to exclude precipitation.

For the heat stress treatment only, wheat plants were irrigated four times, once each at the four stages of jointing, heading, initial grain-filling and middle grain-filling. In the middle of the whole grain-filling stage, wheat plants were stressed under a $38^{\circ} \mathrm{C}$ artificial condition for 3 hours daily for 3 days. This high-temperature treatment was performed using the methods described in previous publications ${ }^{[51]}$ with a slight modification. Namely, a colorless transparent 0.1-mm thick polyethylene plastic was used to make a solar high-temperature shed over the experimental field. The plastic shed could be moved to change the temperature inside. Heat stress was exerted during the peak hours of natural temperature every day from 12:00 to 15:00 pm. A thermometer and hygrometer were placed inside the shed to monitor temperature and humidity.

For the combined stress treatment of drought and heat, irrigation was provided to wheat plants at jointing and heading stages, respectively, and the drought-stress protocol described above was applied from seedling to maturity stage. In the middle stage of grain-filling, the high-temperature stress was applied using the method described above.

For the control treatment, wheat plants grew under natural temperature and sufficient irrigation. Namely, wheat plants were irrigated four times with $900 \mathrm{~m}^{3} \mathrm{hm}^{-2}$, once each during the jointing, heading, early grain-filling, and middle grain-filling stages. The moisture in the upper soil layer (50 $\mathrm{cm}$ in depth) was maintained at $18 \% \pm 1 \%$.

\section{Determining physiological parameters and agronomic traits}

Chlorophyll content (CC) was measured in the upper, middle and lower parts of the flag leaf before and after drought and heat stress using a SPAD-502 chlorophyll meter. For this experiment, five main stems with the same anthesis date were selected and marked for every material in each replicate, and the mean value was calculated. The selection was conducted between 9:00 and 12:00 pm.

Flag leaf water content (LWC) was determined using the samples from the five main stems of each material before and after drought and heat stresses. First, the flag leaf samples were weighed for fresh weight (Wf). Then, the samples were dried in an oven at $60^{\circ} \mathrm{C}$ for $24 \mathrm{~h}$ and weighed for dry weight $(\mathrm{Wd})$. Finally, LWC was calculated using the following formula: LWC $(\%)=(\mathrm{Wf}-$ $\mathrm{Wd}) / \mathrm{Wd} \times 100$. Grain weight per spike (GW) and thousand-grain weight (TGW) were determined at the mature stage of the wheat materials, in which ten main ears were collected from each material in each replicate.

\section{QTL locating}


Microsoft Excel 2010 was used to calculate the phenotypic values. The molecular genetic linkage map used in this study was constructed by Dong et al. ${ }^{[52]}$. The map consisted of 266 marker sites. The total distance of this map was $2187.79 \mathrm{cM}$, and the average distance between the closest two markers was $8.22 \mathrm{cM}$. QTL IciMapping 4.0 positioning software was used to locate the QTLs for drought-tolerant traits in the RILs population. A threshold of LOD > 2.5 was used to determine the existence of QTLs. When the actual LOD value was greater than the threshold LOD, one QTL was considered in that segment. The contribution rates were calculated at $\mathrm{P} \leq 0.05$ and $\mathrm{P} \leq 0.01$ levels, for significant and very significant difference, respectively. The nomenclature for QTL locus is "QTL+ trait + chromosome."

\section{Results}

\section{Related physiological parameters and yield traits of RILs and their parents under stress conditions}

The physiological parameters and yield traits of the RILs populations and their parents were significantly different under different stress conditions in the three environmental sites. The RILs population had wider variation ranges than the parents for all the main physiological parameters and yield traits. The skewness and kurtosis absolute value of each physiological parameter or yield trait was less than or close to 1 , and the $\mathrm{W}$ value was close to 1 , indicating that the physiological parameter and yield trait values of the RILs population are normally distributed and consistent with the inheritance of quantitative loci (Table 1).

\section{QTL mapping of drought-tolerant traits during grain-filling stage}

In total, 30 QTLs were found during the grain-filling stage under the drought stress condition in the three environmental sites. The QTLs controlled CC, LWC, TGW and GW, and were distributed on chromosomes 2A, 3B, 4B, 7B, 3D, 4D and 7D. The phenotypic contribution rate of individual QTLs ranged from 9.38 to $34.2 \%$ (Table 2). Among the 30 QTLs, 8 of the QTLs detected for CC were located on chromosomes 2A, 3B, 4B, 7B, 4D and 3D, and their phenotypic contribution rates ranged from 12.45 to $25.83 \%$. Two synergistic alleles, Qcc-2A.2 and Qcc-7B, were shown to be derived from the drought-tolerant male parent Ningchun27, and their phenotypic contribution rates ranged from 12.45 to $21.46 \%$.

Ten QTLs controlling LWC were detected and they were located on chromosomes 2A, 3B, 7B, 3D, 4D and 7D, and their phenotypic contribution rates ranged from 9.38 to $34.2 \%$. The synergistic alleles of Q/wC-2A.1, Q/wc-2A.2, Q/wC-3B.1, Q/wc-7B.1, Q/wC-3D and QIWC-7D.2 were verified to be from the drought-tolerant male parent Ningchun27, and their phenotypic contribution rates ranged from 9.38 to $30.54 \%$.

Seven QTLs controlling GW were detected to be located on chromosomes 2A, 3B, 4B, 7B and 4D, and their phenotypic contribution rates ranged from 9.75 to $30.89 \%$. The synergistic alleles of $Q g w-3 B .1, Q g w-4 B .2, Q g w-7 B$ and $Q g w-4 D$ were derived from the drought-tolerant male parent Ningchun27, and their phenotypic contribution rates ranged from 9.75 to $25.42 \%$.

Five QTLs controlling TGW were detected, which were located on chromosomes 2A, 3B, 7B and 3D, and their phenotypic contribution rates ranged from 9.75 to $21.22 \%$. The synergistic alleles of Qtgw-2A.3, Qtgw-3B.1, Qtgw-3B.2 and Qtgw-3D were derived from Ningchun27, and their phenotypic contribution rates ranged from 10.76 to $15.87 \%$.

\section{QTL mapping of heat tolerance related traits during grain-filling stage}

Under the thermal stress in the middle stage of grain-filling in the three environmental sites, a total of 41 QTLs controlling CC, LWC, TGW and GW were detected, which are distributed on chromosomes 2A, 3A, 7A, 3B, 4B, 7B, 4D, 5D and 6D. The phenotypic contribution rates of single QTLs ranged from 9.03 to $35.55 \%$ (Table 3).

Out of the 41 QTLs, 14 CC QTLs were detected to be located on chromosomes 2A, 3A, 7A, 3B, 4B, 7B, 4D, 5D and 6D, and the phenotypic contribution rate of single QTLs ranged from 9.35 to $35.55 \%$. The synergistic alleles were derived from the heat-tolerant parent Ningchun4. Ten QTLs controlling LWC were detected to be located on chromosomes 2A, 7A, 2B, 3B, 4B, 7B, 4D and 6D, and the contribution rates of single QTLs ranged from 9.38 to $34.97 \%$. The synergistic alleles were also derived from Ningchun4. Nine QTLs controlling GW were detected on chromosomes 2A, 7A, 3B, 4D, 5D and 6D, and the contribution rates of single QTLs ranged from 9.03 to $27.88 \%$, among which the synergistic alleles on chromosomes $2 A, 7 A, 4 D, 5 D$ and $6 \mathrm{D}$ were confirmed to be derived from the heat-resistant parent Ningchun4, and those on chromosome 3B were derived from the drought-tolerant parent Ningchun27. 
Eight QTLs controlling TGW were detected to be located on chromosomes $2 \mathrm{~A}, 2 \mathrm{~B}, 3 \mathrm{~B}, 4 \mathrm{~B}, 5 \mathrm{D}$ and $6 \mathrm{D}$, and the contribution rates of single QTLs ranged from 9.47 to $32.56 \%$, among which the synergistic alleles on chromosomes 4B, 4D, 5D and 6D were derived from Ningchun4, and the synergistic alleles on chromosomes 2A and 3B were derived from Ningchun27.

\section{QTL mapping of related traits under drought and heat combined stresses during grain-filling period}

Under the combined drought and thermal stresses in the middle stage of grain-filling in the three environmental sites, a total of 46 QTLs controlling CC, LWC, TGW and GW were detected to be distributed on chromosomes 2A, 3A, 4A, 2B, 3B, 4B, 7B, 2D, 3D, 4D and 6D, with single QTL contribution rates ranging from 9.09 to $33.11 \%$ (Table 4). Specifically, 10 QTLs controlling CC were located on chromosomes $2 \mathrm{~A}, 3 \mathrm{~A}, 4 \mathrm{~A}, 2 \mathrm{~B}, 3 \mathrm{~B}, 4 \mathrm{~B}, 3 \mathrm{D}$ and $4 \mathrm{D}$, and the phenotypic contribution rates of single QTLs ranged from 9.09 to $34.35 \%$. The synergistic alleles of $Q c c-2 A .1, Q c c-2 A .2, Q c c-3 A, Q c c-2 B, Q c c-4 B .2$, and $Q c c-4 D$ were derived from Ningchun4, while the synergistic alleles of Qcc-2A.1, Qcc-2B, Qcc-3B.2 and Qcc-3B. 1 were derived from Ningchun27. Thirteen QTLs controlling LWC were located on chromosomes $2 A, 3 A, 2 B, 3 B, 4 B, 7 B, 2 D$ and $4 D$, and the contribution rates of single QTLs ranged from 10.32 to $38.65 \%$.

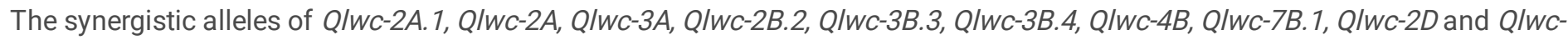
$4 D .1$ were derived from Ningchun4, while the alleles of $Q / W C-2 B .1$, and $Q / W C-7 B .2$ were derived from Ningchun27.

Fourteen QTLs controlling GW were located on chromosomes 2A, 3A, 2B, 3B, 4B, 7B, 3D, 4D and 6D, and the contribution rates of single QTLs ranged from 9.39 to 35.67\%. The synergistic alleles of $Q g w-2 A .2, Q g w-2 A .3, Q g w-4 B .1, Q g w-4 B .3, Q g w-6 D .2$ and $Q g w-$ 6D.3 were derived from Ningchun4, while the alleles of $Q g w-3 A, Q g w-3 B .1, Q g w-3 B .3, Q g w-4 B .2, Q g w-7 B, Q g w-3 D$ and $Q g w-4 D w e r e$ derived from Ningchun27. Nine QTLs controlling TGW were located on chromosomes 2B, 3B, 7B, 2D, 3D, 4D and 6D, and the contribution rates of single QTLs ranged from 8.81 to $27.21 \%$. The synergistic alleles of Qtgw-2D,Qtgw-6D.2 and Qtgw-6D.3 were derived from Ningchun4, while the alleles of $Q \operatorname{tg} w-2 B, Q \operatorname{tg} w-3 B .2, Q \operatorname{tg} w-7 B .2, Q \operatorname{tg} w-3 D$ and $Q \operatorname{tg} w-4 D$ were derived from Ningchun27.

\section{Genetic overlapping of QTLs associated with drought and heat tolerances}

\section{QTL overlapping of drought-tolerant associated traits}

During the grain-filling period in the three environmental sites and 3 years, a total of 30 QTLs were detected under drought treatment (Table 2). Among them, the QTLs QIwC-2A.2 (16E3) for LWC and Qtgw-2A.3 (16E1) for TGW on chromosome 2A were detected to overlap in the interval of barc309-gwm122 (Fig. 1a). The phenotypic contribution rate ranged from 9.75 to $11.34 \%$. The QTLs Qgw3B (15E1 and 16E2) for GW and Qtgw-3B.1 (17E3) for TGW on chromosome 3B overlapped in the interval wmc808-wmc78 (Fig. 1b). These QTLs were detected in all three environmental sites and all three stress treatments and in each of the three years (15E1, 16E1, 16E2, and 17E3), and their phenotypic contribution rates ranged from 11.34 to $25.42 \%$ (Table 2). Moreover, the QTLs Q/Wc-4D (16E1 and 17E2) for LWC and Qgw-4D (15E1) for GW were detected to overlap in the interval of wmc720-wmc331 (Fig. 1c); and the QTLs Q/wC-7B.1 (15E2, 16E3 and 17E1) for LWC, and Qtgw-7B (15E1) for TGW were detected to overlap in the interval wmc83$w m c 276$ (Fig. 1d). Additive effect analysis showed that the synergistic alleles of overlapping QTLs Q/wC-2A.2 and Qtgw-2A.2, and $Q g w-3 B$ and $Q t g w-3 B$ were derived from Ningchun27, these synergistic alleles were closely related to drought-tolerance during grainfilling period. Meanwhile, overlapping QTLs located in wmc720-wmc331 and wmc83-wmc276 were derived from Ningchun4.

\section{QTL overlapping of heat-tolerant associated traits}

A total of 41 QTLs were detected under heat stress conditions during the grain-filling period in the three environmental sites in all three years (Table 3). The QTLs Qcc-2A (15E1) for CC and Qgw-2A (15E1) for GW on chromosome 2A overlapped in the interval of gwm294-wmc644 (Fig. 2a). On chromosome 7B, the QTLs Qcc-7B.1(15E1) for CC, Q/wc-7B.1(15E1, 16E2 and 17E3) for LWC,Qgw7B.1 (15E1) for GW and Qtgw-7B.1 (15E1) for TGW overlapped in the interval of wmc83-wmc276 (Fig. 1d). On chromosome 6D, the QTLs QIWC-6D(15E1) for LWC, Qgw-6D.1 (15E1) for GW, and Qtgw-6D.1 (15E1) for TGW overlapped in the interval of barc196-barc54 (Fig. 2b). Additive effect analysis showed that the synergistic alleles of the three overlapping QTLs were derived from Ningchun4, which is known for its heat tolerance. Their phenotypic contribution rates ranged from 9.03 to $34.97 \%$.

\section{QTL overlapping of combined drought and heat tolerance associated traits}

Two genetic overlapping sites of drought and heat tolerance associated traits were detected on chromosome $2 \mathrm{~A}$ in this study. The QTLs Qcc-2A (15E1, 15E2, and 16E1) for CC and Qgw-2A (15E1, 16E2, and 17E3) for GW were overlapping within gwm294-wmc644 
under combined drought and high-temperature treatment (Fig. 1a and 2a). The QTLs Qcc-2A.1 for CC, Q/wc-2A.2 for LWC, and Qgw2A. 2 for GW were also overlapping within gwm294-wmc644 (Fig. 3a) under combined drought and heat treatment. Their phenotypic contribution rate was 10.12 to $33.0 \%$. These results indicated that the genetic effect of the two overlapping sites was large and the effect of the stress was small. Additive effect analysis showed that the synergistic alleles for CC and TGW under drought stress were derived from Ningchun27. The alleles for CC, LWC and GW under heat stress were derived from Ningchun4, indicating that gwm382-gwm526 and gwm294-wmc644 were closely associated with both drought and heat tolerances.

On chromosome 2B, the QTLs Qcc-2B (15E1) for CC, QIwC-2B (16E3) for LWC, Qgw-2B (15E1) for GW, and Qtgw-2B (15E1) for TGW overlapped in the interval wmc441-wmc317(Fig. 3b), and their phenotypic contribution rates ranged from 13.27 to $25.83 \%$. This result indicated that the genetic effect of the 2 overlapping sites was large and the effect of the stresses was small. Additive effect analysis showed that the synergistic alleles for GW and TGW were derived from Ningchun27, and the synergistic allele for LWC were derived from Ningchun4. The alleles for $\mathrm{CC}$ were from both parents, indicating that the overlapping site within wmc441wmc317 was closely associated with combined drought and heat tolerances.

Under the three stress treatments in all three environments in the 3 years, QTLs Qgw-3B (15E1, 16E2 and 17E1) for GW and Qtgw-3B (16E2) for TGW had overlapping sites detected on chromosome 3B, which overlapped in the interval of wmc808-wmc78 (Fig. 3c). The QTL QIWC-3B (16E2) for LWC, and Qtgw-3B (15E1, 16E2 and 17E3) for TGW overlapped in the interval of wmc787-wmc687, and their phenotypic contribution rates ranged from 11.35 to $29.33 \%$. It is indicated that the genetic effect of the overlapping sites was large and the effect of the stresses was small. Additive effect analysis showed that under drought stress, the synergistic alleles for panicle number and TGW were derived from Ningchun27, and the alleles for LWC under heat stress were derived from Ningchun4. These results indicated that the overlapping site $w m c 808-w m c 78$ was closely associated with drought tolerance, and the overlapping site wmc441-wmc317 was closely associated with both drought and heat tolerances.

Under the same conditions aforementioned, the QTLs Qcc-7B.1 (16E2 and 17E2) for CC, Q/wC-7B.1 (15E1, 16E1 and 17E1) for LWC, and Qtgw-7B (15E1 and 16E1) for TGW on chromosome 7B overlapped in the interval of wmc83-wmc276 (Fig. 1d), and their phenotypic contribution rates ranged from 21.98 to $40.18 \%$. This indicated that the genetic effect of the overlapping sites was large and the effect of the stresses was small. Additive effect analysis showed that the synergistic alleles for CC and GW were derived from Ningchun4, and the alleles for TGW were derived from Ningchun27. The overlapping site wmc83-wmc276 was closely associated with both drought and heat tolerance.

The QTL QIwC-4D (16E2) for LWC, Qgw-4D (15E1) for GW, and Qtgw-4D (15E1) for TGW on chromosome 4D overlapped in the interval of wmc720-wmc331 (Fig. 1c), and their phenotypic contribution rates ranged from 9.75 to $20.32 \%$, indicating that the genetic effect of the overlapping sites was large and the effect of stresses was small. Additive effect analysis showed that the synergistic alleles for GW and TGW were derived from Ningchun27, the synergistic alleles for LWC were derived from Ningchun4, and the overlapping site wmc720-wmc331 was closely associated with both drought and heat tolerance.

\section{Discussion}

In this study, QTL locating of related traits to physiological parameters and yield components was studied under drought and heat stresses in three distinct environments: Yinchuan (E1), Hohhot (E2) and Urumqi (E3). A total of 137 QTL $_{S}$ were detected, among which 30 were detected under drought stress (Table 2), 41 under heat stress (Table 3), 46 under drought and heat combined stresses (Table 4), and 20 under normal conditions (Table 5). Totally, we detected 35 new QTLs, of which 16 were associated with heat tolerance, located on chromosomes 4B, 4D, 5D and 6D; other 19 were associated with combined drought and heat tolerance, located on chromosomes 2A, 3A, 3B and 7B. Our research showed that the QTLs controlling traits related to drought and heat tolerance in wheat were significantly different under different stress conditions, similar to the results from previous reports (Pinto et al. 2010; Tahmasebi et al. 2016). In the 137 QTLs detected in this study, most are independent from each other, and a few QTLs were expressed under different environments and abiotic stresses. For example, the QTLs gwm382-gwm526 on chromosome 2A and wmc83-wmc276 on chromosome 7B were expressed in all three environments in all three years. Therefore, QTL mapping associated with abiotic stress tolerances of wheat should be carried out in different environments under different stresses in different years. It is beneficial to uncover the QTLs that are not sensitive to environmental conditions, and are stably expressed. 
Two overlapping sites for drought tolerance, three overlapping sites for heat tolerance, and four overlapping sites for drought and heat combined tolerances were detected in this investigation. Each overlapping site controlled two to four traits related to drought and heat tolerances. These results indicated that QTLs for drought and heat tolerances are pleiotropic or closely linked on the chromosome. The direction of additive effects of the QTLs indicated by genetic overlapping sites was inconsistent in different environments and years. For example, the overlapping site wmc808-wmc78 on chromosome 3B controlled GW and TGW, but its additive effect was negative, and the synergistic alleles were all from Ningchun27. The overlapping site wmc83-wmc276 on chromosome 7B controlled CC, LWC, GW and TGW, the additive effects were all positive, and the synergistic alleles were from Ningchun4. These two overlapping $Q T L_{S}$ were not sensitive to heat stress and could express stably in response to different environments and stresses. The overlapping site wmc441-wmc317 on chromosome 2B controlled CC, LWC, GW and TGW, and it exhibited positive or negative additive effects. It is noteworthy that the overlapping site gwm294-wmc644 on chromosome $2 \mathrm{~A}$ was detected under the conditions of drought stress, heat stress, and combined drought and heat stress for two years in three environments. The allele controlling CC was derived from drought-resistant parent Ningchun27, and the allele controlling LWC, GW and TGW was derived from heat-resistant parent Ningchun4. Therefore, we inferred that the synergistic alleles could be from both parents and played roles in both drought and heat tolerances. Therefore, it is important to uncover the overlapped QTLs associated with drought- and heat-tolerance that are not sensitive to environmental conditions and can express stably. Using these overlapping sites as markers, it will be possible to carry out gene pyramiding breeding and simultaneously improve multi-tolerances in wheat via marker-assisted selection.

In terms of the genetic mechanisms of drought and heat tolerances in wheat, previous investigators have begun meaningful research. Regarding the gene localization of drought tolerance, the results in this study are somewhat consistent with those in previous studies. For example, several QTLs related to drought tolerance in wheat were detected on chromosomes $2 \mathrm{~A}$ (Xu et al 2017), 7B (Czyczyło-Mysza et al. 2011; Xu et al. 2017) and 4B (Kadam et al. 2012; Tahmasebi et al. 2016). In this study, the QTLs controlling CC were detected on chromosome 3B, and QTLs controlling TGW were detected on chromosomes 2A, 3B, 4D and 6D, which were consistent with the results reported by Tahmasebi et al. (2016). As for heat tolerance gene localization in wheat, related QTLs were detected on chromosomes 2A, 2B, 3B, 7A and 7B (Vijayalakshmi et al. 2010; Pinto et al. 2010; Mason et al. 2012; Paliwal et al. 2012; Li et al. 2013). Variations from previous results are due to the differences in experimental materials, identification methods, and QTL positioning tools application. Because of the large amount of genomic characteristics and the complexity of stress tolerance inheritance in wheat, further studies are needed on QTL mapping of stress tolerance in multiple environments under different stresses in order to develop more functional molecular markers on stress tolerance of this crop.

Wheat is sensitive to drought and heat stress during the grain-filling period (Barlow et al. 2015; Ni et al. 2018). Therefore, enhancement of drought and heat tolerance during this period is very important for sustaining acceptable wheat yields. This study identified the overlapping sites barc309-gwm122 that affected drought tolerance during grain-filling. The synergistic alleles for drought tolerance were derived from Ningchun27, the drought-tolerant parent. The overlapping sites barc196-barc54 affecting heat tolerance during grain-filling were also identified, and the synergistic alleles for this trait were derived from the heat-tolerant parent, Ningchun4. The genetic distance between these two overlapping sites is small and the phenotypic contribution rate is large. By gene pyramiding using marker-assisted selection for these two sites, it is possible to enhance the drought and heat tolerances of wheat during the grain-filling period. The overlapping sites gwm294-wmc644 affecting drought and heat combined tolerances during this period were identified and the synergistic alleles were shown to be derived from both the drought-tolerant and heattolerant parents. The genetic distance between these two overlapping sites is also small and the phenotypic contribution rate is also large. By using marker-assisted selection for drought- and heat-tolerance overlapping sites, it will be possible to increase the efficiency of breeding new wheat varieties that are tolerant to drought and heat.

\section{Declarations}

\section{Acknowledgements}

We sincerely thank Mrs. Jun Ye at the Crop Research Institute of the Academy of Agricultural and Pastoral Sciences, Hohhot, Inner Mongolia, and Chaowu Zeng at the Crop Research Institute of the Xinjiang Academy of Agricultural Sciences, Urumqi, Xinjiang, for their kind coordination and management of experiments during the growing season. 


\section{Funding}

This research was financially supported by the National Natural Science Foundation of China $(31460345,31960428)$, the Department of Science and Technology of Ningxia Hui Autonomous Region of China (2018NYYZ02), and the Third Batch of Ningxia Youth Talents Supporting Program (TJGC2018025).

\section{Conflicts of interest}

The authors declare that they have no conflict of interest.

\section{Ethics approval}

This article does not contain any studies with human participants or animals performed by any of the authors.

\section{Authors' contributions}

The experiment was conceived by JLD, HBB, LS and XGY. HBB, YXZ and JLD examined the botanical and agronomic traits. XLL, SHL and JH determined the physiological parameters. HBB and JLD analyzed the experimental data. HBB, JLD and LS performed the QTL analysis and constructed the chromosomal linkage maps. The manuscript was drafted by JLD, HBB, LS and XGY, and corrected and approved by all authors.

\section{References}

Barlow KM, Christy BP, O'Leary GJ, Riffkin PA, Nuttallb JG (2015) Simulating the impact of extreme heat and frost events on wheat crop production: A review. Field Crops Res 171:109-119

Bennett D, Reynolds M, Mullan D, Izanloo A, Kuchel H, Langridge P, Schnurbusch T (2012) Detection of two major grain yield QTL in bread wheat (Triticum aestivum L.) under heat, drought and high yield potential environments. Theor Appl Genet 125:1473-1485

Budak H, Hussain B, Khan Z, Ozturk NZ, Ullah N (2015) From genetics to functional genomics: improvement in drought signaling and tolerance in wheat. Front. Plant Sci 11(6):1012

Caverzan A, Casassola A, Brammer SP (2016) Antioxidant responses of wheat plants under stress. Genet Mol Biol 39(1):1-6

Czyczyło-Mysza I, Marcińska I, Skrzypek E, Chrupek M, Grzesiak S, Hura T,Stojałowski S, Myś ków B, Milczarski P, Quarrie S (2011) Mapping QTLs for yield components and chlorophyll a fluorescence parameters in wheat under three levels of water availability. Plant Genet Resour 9:291-295

Dashti H, Yazdisamadi B, Bihamta Naghavi MR, Quarrie S (2007) QTL analysis for drought resistance in wheat using doubled haploid lines. Int J Agric Biol 9:98-101

Dong J, Li S, Hui H, Kong D (2008) Effects of dry and high temperature stress on different wheat genotype yield trait. (in Chinese with English abstract) Acta Agri Boreali -Occident Sin, 17(6):63-66

Dong J, Bai H, Zhu Y, Zhao P, Lu X, Li S, Wang Z (2015) Construction of wheat linkage map using a set of spring wheat recombinant inbred lines and different SSR markers. (in Chinese with English abstract). Sci Tech Review 33(7):84-89

Grigorova B, Vaseva I, Demirevska K, Feller U (2011) Combined drought and heat stress in wheat: changes in some heat shock proteins. Biol Plant 55(1), 105-111

He D, Wang J,Pan Z, Dai T, Wang E, Zhang J (2017) Changes in wheat potential productivity and drought severity in Southwest China. Theor Appl Climatol 130:477-486

He G, Xu J, Wang Y, Liu J, Li P, Chen M, Ma Y, Xu Z (2016) Drought-responsive WRKY transcription factor genes TaWRKY1 and TaWRKY33 from wheat confer drought and/or heat resistance in Arabidopsis. BMC Plant Biol 16:116 
Hu X, Rocheleau H, McCartney C, Biselli C, Bagnaresi P, Balcerzak M, Fedak G, Yan Z, Valè G, Khanizadeh S, Ouellet T (2019) Identification and mapping of expressed genes associated with the 2DL QTL for fusarium head blight resistance in the wheat line Wuhan 1. BMC Genet 20:47

Hu Y, Liu Y, Tang H, Xu Y, Pan J (2014) Contribution of drought to potential crop yield reduction in a wheat-maize rotation region in the north china plain. J Integr Agr 13(7):1509-1519

Ilyas M, llyas N, Arshad M, Kazi AG(2014) QTL mapping of wheat doubled haploids for chlorophyll content and chlorophyll fluorescence kinetics under drought stress imposed at anthesis stage. Pak J Bot. 46, 1889-1897

Johnson SM, Lim FL, Finkler A Fromm H, Slabas AR, Knight MR (2014) Transcriptomic analysis of Sorghum bicolor responding to combined heat and drought stress. BMC Genom 15:456

Kadam S, Singh K, Shukla S, Goel S, Vikram P, Pawar V, Gaikwad K, Khanna-Chopra R, Singh N (2012) Genomic associations for drought tolerance on the short arm of wheat chromosome 4B. Funct Integr Genom 12:447-464

Kirigwi FM, Van Ginkel M, Brown-Guedira G, Gill BS, Paulsen GM, Fritz AK (2007) Markers associated with a QTL for grain yield in wheat under drought. Mol Breed 20:401-413

Kumari S, Jaiswal V, Mishra VK, Paliwal R, Balyan HS, Gupta PK (2018) QTL mapping for some grain traits in bread wheat (Triticum aestivum L.). Physiol Mol Biol Plant 24(5):909-920

Lawas LMF, Zuther E, Jagadish SVK, Hincha DK (2018) Molecular mechanisms of combined heat and drought stress resilience in cereals. Curr Opin Plant Biol 45:212-217

Li S, Chang X, Wang C Jing R (2013) Mapping QTL for heat tolerance at grain filling Stage in common wheat. (in Chinese with English abstract) Sci Agri Sin 46(10):2119-2129

Liu C, Sukumaran S, Claverie E, Sansaloni C, Dreisigacker S, \& Reynolds M (2019) Genetic dissection of heat and drought stress QTLs in phenology-controlled synthetic-derived recombinant inbred lines in spring wheat. Mol Breed 39(3)

Liu K, Xu H, Liu G, Guan P, Zhou X, Peng H, Yao Y,Ni Z, Sun Q, Du J (2018) QTL mapping of flag leaf-related traits in wheat (Triticum aestivum L.). Theor Appl Genet 131(8):839-849

Liu L, Yuan CY, Wang MN, See DR, Zemetra RS, Chen XM (2019) QTL analysis of durable stripe rust resistance in the North American winter wheat cultivar Skiles. Theor Appl Genet 132(6):1677-1691

Liu Z, Xin M, Qin J Peng H, Ni Z, Yao Y, Sun Q (2015) Temporal transcriptome profiling reveals expression partitioning of homeologous genes contributing to heat and drought acclimation in wheat (Triticum aestivum L.). BMC Plant Biol 15:152

Maccaferri M, Sanguineti MC, Corneti S, Ortega JLA, Salem MB, Bort J, DeAmbrogio E, del Moral LFG, Demontis A, El-Ahmed A, Maalouf F, Machlab H, Martos V, Moragues M, Motawaj J, Nachit M, Nserallah N, Ouabbou H, Royo C, Slama A, Tuberosa R (2008) Quantitative trait loci for grain yield and adaptation of durum wheat (Triticum durum Desf.) across a wide range of water availability. Genetics 178:489-511

Mason RE, Mondal S, Beecher FW, Pacheco A, Jampala B, Ibrahim AMH, Hays DB (2010) QTL associated with heat susceptibility index in wheat (Triticum aestivum L.) under short-term reproductive stage heat stress. Euphytica 174:423-436

Merchuk-Ovnat L, Fahima T, Krugman T, Saranga Y (2016) Ancestral QTL alleles from wild emmer wheat improve grain yield, biomass and photosynthesis across environments in modern wheat. Plant Sci 251:23-34

Miles C, Wayne M (2008) Quantitative trait locus (QTL) analysis. Nature Education 1(1):208

Mohammadi V, Zali AA, Bihamta MR(2008) Mapping QTLs for heat tolerance in wheat. J Agric Sci Technol 10, 261-267

Page $10 / 23$ 
Muqaddasi QH, Jayakodi M, Börner A, Röder MS (2019) Identification of consistent QTL with large effect on anther extrusion in doubled haploid populations developed from spring wheat accessions in German Federal ex situ Genebank. Theor Appl Genet 132:3035-3045

Mwadzingeni L, Shimelis H, Dube E, Laing MD, Tsilo TJ (2016) Breeding wheat for drought tolerance: Progress and technologies. J Integr Agri 15(5):935-943

Mwadzingeni L, Figlan S, Shimelis H, Mondal S, Tsilo TJ (2017) Genetic resources and breeding methodologies for improving drought tolerance in wheat. J Crop Improve 31(5):648-672

Ni Z, Li H, Zhao Y, Peng H, Hu Z, Xin M, Sun Q (2018) Genetic improvement of heat tolerance in wheat: Recent progress in understanding the underlying molecular mechanisms. Crop J 6:32-41

Ogbonnaya FC, Rasheed A, Okechukwu EC Jighly A, Makdis F, Wuletaw T, Hagras A, Uguru Ml, Agbo CU (2017) Genome-wide association study for agronomic and physiological traits in spring wheat evaluated in a range of heat prone environments. Theor Appl Genet 130:1819-1835

Paliwal R, Röder MS, Kumar U Srivastava JP, Joshi AK (2012) QTL mapping of terminal heat tolerance in hexaploid wheat ( $T$. aestivum L.). Theor Appl Genet, 125:561-575

Peleg Z, Fahima T, Krugman T Abbo S, Yakir D, Korol AB, Saranga Y (2009) Genomic dissection of drought resistance in durum wheat $\times$ wild emmer wheat recombinant inbreed line population. Plant Cell Environ 32:758-779

Pinto RS, Reynolds MP, Mathews KL, Mclntyre CL, Olivares-Villegas JJ, Chapman SC (2010) Heat and drought adaptive QTL in a wheat population designed to minimize confounding agronomic effects. Theor Appl Genet 121:1001-1021

Pinto RS, Reynolds MP (2015) Common genetic basis for canopy temperature depression under heat and drought stress associated with optimized root distribution in bread wheat. Theor Appl Genet 128:575-585

Quarrie SA, Steed A, Calestani C, Semikhodskii A, Lebreton C, Chinoy C, Steele N, Pljevljakusić D, Waterman E, Weyen J, Schondelmaier J, Z. Habash D, Farmer P, Saker L, ClarksonDT, Abugalieva A, Yessimbekova M, Turuspekov Y, Abugalieva M, Tuberosa R, Sanguineti M-C, Hollington PA, Aragués R, Royo A, Dodig D (2005) A high-density genetic map of hexaploid wheat (Triticum aestivum L.) from the cross Chinese Spring $\times$ SQ1 and its use to compare QTLs for grain yield across a range of environments. Theor Appl Genet 110:865-880

Shirdelmoghanloo H, Taylor JD, Lohraseb I, Rabie h, Brien C, Timmins A, Martin P, Mather DE, Emebiri L, Collins NC(2016) A QTL on the short arm of wheat (Triticum aestivum L.) chromosome 3B affects the stability of grain weight in plants exposed to a brief heat shock early in grain filling. BMC Plant Biol 16:100

Sukumaran S, Reynolds MP, Sansaloni C (2018) Genome-wide association analyses identify QTL hotspots for yield and component traits in durum wheat grown under yield potential, drought, and heat stress environments. Front Plant Sci 9:81

Szucs A, Jager K, Jurca ME et al. (2010) Histological and microarray analysis of the direct effect of water shortage alone or combined with heat on early grain development in wheat (Triticum aestivum). Physiol Plant 140:174-188

Tahmasebi S, Heidari B, Pakniyat H, Mclntyre CL (2016) Mapping QTLs associated with agronomic and physiological traits under terminal drought and heat stress conditions in wheat (Triticum aestivum L.). Genome, 59:1-20

Tricker PJ, ElHabti A, Schmidt J Fleury D (2018) The physiological and genetic basis of combined drought and heat tolerance in wheat. J Exp Bot 69(13):3195-3210

Vijayalakshmi K, Fritz AK, Paulsen GM, Bai G, Pandravada S, Gill BS(2010). Modeling and mapping QTL for senescence-related traits in winter wheat under high temperature. Mol. Breed 26, 163-175 
Wan Y, King R, Mitchell RAC, Hassani-Pak K, Hawkesford MJ (2017). Spatiotemporal expression patterns of wheat amino acid transporters reveal their putative roles in nitrogen transport and responses to abiotic stress. Sci Rep 7(1):5461

Xu Y, Li S, Li L, Ma F, Fu X, Shi Z, Xu H, Ma P, An D (2017) QTL mapping for yield and photosynthetic related traits under different water regimes in wheat. Mol Breed 37:34

Yang D, Jing R, Chang X, Li W (2007) Identification of quantitative trait loci and environmental interactions for accumulation and remobilization of water-soluble carbohydrates in wheat (Triticum aestivum L.) stems. Genetics 176:571-584

Ye X, Li J, Cheng Y, Yao F, Long L, Wang Y, Wu Y, Li J, Wang J, Jiang Q, Kang H, Li W, Qi P, Lan X, Ma J, Liu Y, Jiang Y, Wei Y, Chen X, Liu C, Zheng Y, Chen G (2019) Genome-wide association study reveals new loci for yield-related traits in Sichuan wheat germplasm under stripe rust stress. BMC Genom 20:640

Zhang H, Li W, Mao X, Jing R, Jia H (2016) Differential activation of the wheat SnRK2 family by abiotic stresses. Front Plant Sci $7: 420$

Zhang J, Yang W, Yue J, Liu Y, Pei D, Wang H (2019) The responses of wheat autophagy and ATG8 family genes to biotic and abiotic stresses. J Plant Growth Regul doi:https://doi.org/10.1007/s00344-019-10027-w

\section{Tables}

Table 1 Main physiological index and yield traits in the RILs population under different stresses 


\begin{tabular}{|c|c|c|c|c|c|c|c|c|}
\hline \multirow[t]{2}{*}{ Stress types } & \multirow[t]{2}{*}{ Traits } & \multirow[t]{2}{*}{ Environments } & \multicolumn{2}{|c|}{ Parents } & \multicolumn{4}{|l|}{ RIL } \\
\hline & & & Ningchun4 & Ningchun27 & Range & Skewness & Kurtosis & W test \\
\hline \multirow[t]{13}{*}{ Drought } & $\mathrm{CC}$ & E1 & 52.92 & 45.41 & $17.71-57.10$ & -0.65 & 0.75 & 0.95 \\
\hline & & E2 & 50.42 & 42.91 & $24.60-59.00$ & 0.56 & 0.27 & 0.96 \\
\hline & & E3 & 47.47 & 46.62 & $14.18-52.08$ & 0.84 & 0.60 & 0.96 \\
\hline & LWC & E1 & 68.71 & 67.44 & $17.36-79.40$ & 0.37 & 0.18 & 0.97 \\
\hline & & E2 & 64.78 & 67.63 & 26.34-79.52 & 0.22 & 0.62 & 0.96 \\
\hline & & E3 & 61.02 & 64.92 & $20.45-61.87$ & 0.70 & 0.76 & 0.89 \\
\hline & GW & E1 & 1.56 & 2.21 & $0.93-3.30$ & 0.13 & 0.79 & 0.98 \\
\hline & & E2 & 1.63 & 1.60 & $1.16-2.80$ & 0.84 & 0.80 & 0.95 \\
\hline & & E3 & 1.54 & 1.42 & $0.58-3.10$ & 0.99 & 0.69 & 0.94 \\
\hline & TGW & E1 & 44.01 & 45.68 & $31.36-59.90$ & 0.60 & 0.87 & 0.88 \\
\hline & & E2 & 58.14 & 55.79 & 42.59-59.33 & 0.11 & 0.64 & 0.96 \\
\hline & & E3 & 43.07 & 46.86 & $21.74-55.70$ & 0.48 & 0.65 & 0.97 \\
\hline & & Mean & 41.27 & 40.71 & 39.14 & 0.43 & 0.63 & 0.95 \\
\hline \multirow[t]{13}{*}{ Heat } & $\mathrm{CC}$ & $\mathrm{E} 1$ & 50.75 & 43.28 & $13.42-57.84$ & 0.85 & 0.53 & 0.89 \\
\hline & & E2 & 49.47 & 48.66 & $19.53-59.36$ & 0.82 & 0.75 & 0.95 \\
\hline & & E3 & 48.14 & 48.62 & $4.94-52.11$ & 0.81 & 0.51 & 0.91 \\
\hline & LWC & E1 & 66.17 & 61.94 & $15.43-71.37$ & 0.91 & 1.06 & 0.88 \\
\hline & & E2 & 67.22 & 66.62 & 19.28-77.86 & 0.63 & 0.53 & 0.96 \\
\hline & & E3 & 61.23 & 64.31 & $16.32-70.25$ & 0.16 & 0.85 & 0.90 \\
\hline & GW & E1 & 1.51 & 1.64 & $0.91-3.42$ & 0.17 & 0.81 & 0.97 \\
\hline & & E2 & 1.37 & 1.49 & $1.22-3.32$ & 0.52 & 0.34 & 0.96 \\
\hline & & E3 & 1.45 & 2.03 & $0.83-2.91$ & 0.05 & 0.21 & 0.97 \\
\hline & TGW & E1 & 41.78 & 42.72 & $30.28-61.32$ & 0.45 & 0.04 & 0.97 \\
\hline & & E2 & 42.68 & 44.08 & $31.13-65.67$ & 0.16 & 0.04 & 0.98 \\
\hline & & E3 & 43.38 & 48.78 & 28.74-63.33 & 0.01 & 0.78 & 0.98 \\
\hline & & Mean & 39.60 & 39.51 & 38.16 & 0.46 & 0.54 & 0.94 \\
\hline \multirow{8}{*}{$\begin{array}{l}\text { Combined } \\
\text { drought and heat }\end{array}$} & $\mathrm{CC}$ & $\mathrm{E} 1$ & 40.37 & 43.35 & $12.94-56.47$ & 0.93 & 0.31 & 0.91 \\
\hline & & E2 & 47.18 & 45.56 & $17.32-53.60$ & 0.66 & 0.22 & 0.94 \\
\hline & & E3 & 40.04 & 45.74 & $14.32-52.33$ & 0.37 & 0.80 & 0.94 \\
\hline & LWC & E1 & 63.94 & 60.32 & $24.74-79.43$ & 0.37 & 0.18 & 0.97 \\
\hline & & E2 & 65.83 & 60.12 & 17.75-78.75 & 0.87 & 0.98 & 0.95 \\
\hline & & E3 & 58.11 & 63.14 & $15.43-65.89$ & 0.16 & 1.05 & 0.90 \\
\hline & GW & $\mathrm{E} 1$ & 1.47 & 1.52 & $0.35-2.74$ & 0.13 & 0.99 & 0.98 \\
\hline & & E2 & 1.15 & 1.38 & $1.17-3.21$ & 0.80 & 0.97 & 0.94 \\
\hline
\end{tabular}

Page 13/23 


\begin{tabular}{|cllllllll|} 
& E3 & 1.34 & 1.84 & $0.56-2.84$ & 0.70 & 0.84 & 0.95 \\
\hline TGW & E1 & 39.56 & 41.03 & $24.13-56.48$ & 0.90 & 0.97 & 0.88 \\
\hline & E2 & 40.59 & 44.81 & $37.24-64.71$ & 0.35 & 0.45 & 0.97 \\
\hline & E3 & 39.39 & 45.67 & $20.96-55.34$ & 0.20 & 0.30 & 0.98 \\
\hline & Mean & 36.58 & 37.87 & 36.24 & 0.54 & 0.67 & 0.94 \\
\hline
\end{tabular}

Note: CC, chlorophyll content; LWC, leaf water content; GW, grain weight per spike; TGW, thousand-grain weight; E1, Yinchuan 20152017; E2, Hohhot 2015-2017; E3, Urumqi 2015-2017.

Table 2 QTL mapping of drought-resistant traits in multiple years and locations 


\begin{tabular}{|c|c|c|c|c|c|c|c|c|c|}
\hline \multirow[t]{2}{*}{ Traits } & \multirow[t]{2}{*}{ QTL } & \multirow[t]{2}{*}{ Environments } & \multirow[t]{2}{*}{ Marker interval } & \multicolumn{2}{|c|}{ Variation (\%) } & \multicolumn{3}{|c|}{ Additive effect } & \multirow[b]{2}{*}{2017} \\
\hline & & & & 2015 & 2016 & 2017 & 2015 & 2016 & \\
\hline \multirow[t]{7}{*}{$\mathrm{CC}$} & $Q c c-2 A .1$ & $15 \mathrm{E} 1$ & gwm382-gwm526 & 23.06 & & & 1.56 & & \\
\hline & Qcc-2A.2 & 15E2, 16E1 & gwm294-wmc644 & 13.53 & 21.46 & & -1.58 & -1.45 & \\
\hline & $Q c c-3 B$ & 17E1 & gwm376-barc164 & & & 16.54 & & & 2.08 \\
\hline & $Q c c-3 B .1$ & $16 \mathrm{E} 2$ & wmc808-wmc78 & & 19.04 & & & -3.121 & \\
\hline & $Q c c-4 B$ & 15E2, 16E1 & gwm495-gwm375 & 13.15 & 12.51 & & 1.37 & 1.38 & \\
\hline & $Q c c-7 B$ & 15E1 & barc140-gwm297 & 12.45 & & & -1.13 & & \\
\hline & $Q C C-3 D$ & $16 \mathrm{E} 3$ & gpw322-gpw4451 & & 25.51 & & & 8.73 & \\
\hline \multirow[t]{10}{*}{ LWC } & QIwc-2A.1 & $15 \mathrm{E} 1$ & gwm425-wmc522 & 11.93 & & & -1.25 & & \\
\hline & Q/wc-2A.2 & 16E3 & barc309-gwm122 & & 11.34 & & & -6.24 & \\
\hline & Q/wc-3B.1 & 15E1 & barc164-gwm108 & 9.38 & & & -0.76 & & \\
\hline & Q/wc-3B.2 & 17E1 & gwm376-barc164 & & & 16.54 & & & 2.08 \\
\hline & QIwc-7B.1 & 15E2, 16E3, 17E1 & wmc83-wmc276 & 26.80 & 21.98 & 30.81 & 15.11 & -20.94 & 11.69 \\
\hline & QIwc-7B.2 & 17E3 & cfa2106-gwm333 & & & 34.20 & & & 12.26 \\
\hline & QIwC-3D & 15E1 & wmc552-gpw4136 & 30.54 & & & -1.40 & & \\
\hline & Q/wC-4D & 16E1, 17E2 & wmc720- wmc331 & & 14.95 & 20.32 & & 1.04 & 1.89 \\
\hline & Q/wc-7D.1 & 17E3 & barc214-cfd46 & & & 12.70 & & & 4.71 \\
\hline & Q/WC-7D.2 & 15E1 & gwm44-gdm67 & 10.38 & & & -0.81 & & \\
\hline \multirow[t]{7}{*}{ GW } & $Q g w-2 A .1$ & 16E3, 17E2 & gwm382-gwm526 & & 13.44 & 30.89 & & 0.15 & 1.73 \\
\hline & $Q g w-2 A .2$ & 15E1, 16E2, 17E3 & gwm294-wmc644 & 19.40 & 11.83 & 13.13 & 0.17 & 0.15 & 0.10 \\
\hline & $Q g w-3 B .1$ & 15E1,16E2 & wmc808-wmc78 & 13.53 & 25.42 & & -0.21 & -0.31 & \\
\hline & $Q g w-4 B .1$ & 15E1 & gwm251-wmc652 & 10.99 & & & 0.15 & & \\
\hline & $Q g w-4 B .2$ & 15E1,16E3 & barc174-wmc657 & & 18.71 & 10.36 & & -0.25 & -0.12 \\
\hline & $Q g w-7 B$ & 15E1 & barc176-gwm400 & 11.02 & & & -0.15 & & \\
\hline & $Q g w-4 D$ & 15E1 & wmc720-wmc331 & 9.75 & & & -1.34 & & \\
\hline \multirow[t]{5}{*}{ TGW } & Qtgw-2A.3 & $16 \mathrm{E} 1$ & barc309-gwm122 & & 10.76 & & & -1.96 & \\
\hline & Qtgw-3B.1 & 17E3 & wmc808-wmc78 & & & 15.87 & & & -2.23 \\
\hline & Qtgw-3B.2 & 17E3 & wmc787-wmc687 & & & 15.13 & & & -2.50 \\
\hline & $Q \operatorname{tg} w-7 B$ & 15E1 & wmc83-wmc276 & 27.22 & & & 5.83 & & \\
\hline & $Q \operatorname{tg} w-3 D$ & 15E1 & gpw4451-barc323 & 13.18 & & & -1.85 & & \\
\hline
\end{tabular}

Note: CC, chlorophyll content; LWC, leaf water content; GW, grain weight per spike; TGW, thousand-grain weight; E1, Yinchuan; E2, Hohhot; E3, Urumqi.

Table 3 QTL mapping of heat tolerance-related traits in multiple locations in three different years 


\begin{tabular}{|c|c|c|c|c|c|c|c|c|c|c|}
\hline \multirow[t]{2}{*}{ Traits } & \multirow[t]{2}{*}{ QTL } & \multirow[t]{2}{*}{ Environments } & \multirow{2}{*}{$\begin{array}{l}\text { Marker } \\
\text { interval }\end{array}$} & \multicolumn{2}{|c|}{ Variation (\%). } & \multicolumn{5}{|c|}{$\underline{\text { Additive effect }}$} \\
\hline & & & & 2015 & 2016 & 2017 & 2015 & 2016 & 2017 & \\
\hline \multirow[t]{16}{*}{$\mathrm{CC}$} & $\begin{array}{l}Q c C^{-} \\
2 A\end{array}$ & 15E1 & & $\begin{array}{l}\text { gwm294- } \\
\text { wmc644 }\end{array}$ & 18.22 & & & 1.62 & & \\
\hline & $\begin{array}{l}Q c c^{-} \\
2 A .3\end{array}$ & 15E1 & & $\begin{array}{l}\text { wmc644- } \\
\text { gwm558 }\end{array}$ & 30.97 & & & 10.59 & & \\
\hline & $\begin{array}{l}Q c C^{-} \\
2 A .4\end{array}$ & 15E1 & & $\begin{array}{l}\text { gwm425- } \\
\text { wmc522 }\end{array}$ & 12.68 & & & 1.43 & & \\
\hline & $\begin{array}{l}Q c C^{-} \\
2 A .5\end{array}$ & 16E1 & & $\begin{array}{l}\text { gwm356- } \\
\text { gwm294 }\end{array}$ & & 13.89 & & & 3.68 & \\
\hline & $\begin{array}{l}Q c c^{-} \\
2 A .6\end{array}$ & 16E3, 17E3 & & $\begin{array}{l}\text { wmc794- } \\
\text { gwm382 }\end{array}$ & & 32.57 & 21.55 & & 11.37 & 3.28 \\
\hline & $\begin{array}{l}Q c c^{-} \\
3 A\end{array}$ & 16E3,17E1 & & $\begin{array}{l}\text { gwm155- } \\
\text { gwm480 }\end{array}$ & & 9.39 & 10.38 & & 3.36 & 1.31 \\
\hline & $\begin{array}{l}Q c c^{-} \\
7 A\end{array}$ & 17E1 & & $\begin{array}{l}\text { wmc603- } \\
\text { wmc809 }\end{array}$ & & & 34.16 & & & 2.04 \\
\hline & $\begin{array}{l}Q c c- \\
2 B\end{array}$ & 16E1 & & $\begin{array}{l}\text { wmc441- } \\
\text { wmc317 }\end{array}$ & & 16.87 & & & 3.15 & \\
\hline & $\begin{array}{l}Q c c^{-} \\
3 B .1\end{array}$ & 15E1, 16E1 & & $\begin{array}{l}\text { wmc808- } \\
\text { wmc78 }\end{array}$ & 14.15 & 19.04 & & -1.55 & -3.12 & \\
\hline & $\begin{array}{l}Q c c- \\
4 B .1\end{array}$ & 15E1 & & $\begin{array}{l}\text { barc20- } \\
\text { gwm495 }\end{array}$ & 12.75 & & & 1.35 & & \\
\hline & $\begin{array}{l}Q c c- \\
4 B .2\end{array}$ & 17E1 & & $\begin{array}{l}\text { gpw7521- } \\
\text { wmc710 }\end{array}$ & & & 12.52 & & & 1.33 \\
\hline & $\begin{array}{l}Q c c- \\
7 B\end{array}$ & 15E1 & & $\begin{array}{l}\text { wmc83- } \\
\text { wmc276 }\end{array}$ & 25.14 & & & 4.47 & & \\
\hline & $\begin{array}{l}Q c c^{-} \\
4 D\end{array}$ & $16 \mathrm{E} 3$ & & $\begin{array}{l}\text { wmc825- } \\
\text { barc288 }\end{array}$ & & 35.55 & & & 6.78 & \\
\hline & $\begin{array}{l}Q c c^{-} \\
5 D\end{array}$ & 15E1 & & $\begin{array}{l}\text { cfd67- } \\
\text { cfd40 }\end{array}$ & 18.28 & & & 1.75 & & \\
\hline & $\begin{array}{l}Q c c- \\
6 D .1\end{array}$ & $16 \mathrm{E} 2$ & & $\begin{array}{l}\text { cfd76- } \\
\text { cfd188 }\end{array}$ & & 11.24 & & & 1.74 & \\
\hline & $\begin{array}{l}Q c c- \\
6 D .2\end{array}$ & 17E1 & & $\begin{array}{l}\text { gpw4308- } \\
\text { cfd76 }\end{array}$ & & & 14.51 & & & 1.45 \\
\hline \multirow[t]{7}{*}{ LWC } & $\begin{array}{l}\text { Qlwc- } \\
2 A .3\end{array}$ & 15E1 & & $\begin{array}{l}\text { wmc296- } \\
\text { gwm425 }\end{array}$ & 11.14 & & & 1.98 & & \\
\hline & $\begin{array}{l}\text { Q/WC } \\
-7 A\end{array}$ & $16 \mathrm{E} 1$ & & $\begin{array}{l}\text { barc121- } \\
\text { wmc607 }\end{array}$ & & 10.68 & & & 0.92 & \\
\hline & $\begin{array}{l}\text { QlWC } \\
-3 B\end{array}$ & $16 \mathrm{E} 2$ & & $\begin{array}{l}\text { wmc787- } \\
\text { wmc687 }\end{array}$ & & 19.99 & & & 1.35 & \\
\hline & $\begin{array}{l}\text { Qlwc- } \\
7 B .1\end{array}$ & 15E1, 16E3, 17E2 & & $\begin{array}{l}\text { wmc83- } \\
\text { wmc276 }\end{array}$ & 34.97 & 33.73 & 25.38 & 18.42 & 7.78 & 6.96 \\
\hline & $\begin{array}{l}\text { Qlwc- } \\
7 B .3\end{array}$ & 16E1 & & $\begin{array}{l}\text { gwm333- } \\
\text { wmc83 }\end{array}$ & & 32.45 & & & 18.43 & \\
\hline & $\begin{array}{l}\text { Q/WC- } \\
4 D\end{array}$ & 15E1, 17E2 & & $\begin{array}{l}\text { wmc720- } \\
\text { wmc331 }\end{array}$ & 11.35 & & 12.08 & 1.99 & & 1.60 \\
\hline & $\begin{array}{l}\text { Qlwc- } \\
4 D .1\end{array}$ & 15E1, 16E2 & & $\begin{array}{l}\text { barc288- } \\
\text { psp3103 }\end{array}$ & 11.82 & 15.31 & & 2.23 & 1.24 & \\
\hline
\end{tabular}

Page 16/23 


\begin{tabular}{|c|c|c|c|c|c|c|c|c|c|}
\hline & $\begin{array}{l}\text { Q/WC- } \\
6 D\end{array}$ & $15 \mathrm{E} 1$ & $\begin{array}{l}\text { barc196- } \\
\text { barc54 }\end{array}$ & 9.38 & & & 1.40 & & \\
\hline \multirow[t]{9}{*}{ GW } & $\begin{array}{l}Q g W^{-} \\
2 A .2\end{array}$ & $15 \mathrm{E} 1$ & $\begin{array}{l}\text { gwm294- } \\
\text { wmc644 }\end{array}$ & 15.86 & & & 0.17 & & \\
\hline & $\begin{array}{l}\text { Qgw- } \\
7 A\end{array}$ & $15 \mathrm{E} 1$ & $\begin{array}{l}\text { wmc603- } \\
\text { wmc809 }\end{array}$ & 15.46 & & & 0.16 & & \\
\hline & $\begin{array}{l}\text { Qgw- } \\
3 B .1\end{array}$ & 15E1, 16E2, 17E3 & $\begin{array}{l}\text { wmc808- } \\
\text { wmc78 }\end{array}$ & 21.44 & 27.88 & 22.78 & -0.17 & -0.23 & -0.17 \\
\hline & $\begin{array}{l}\text { QgW- } \\
3 B .2\end{array}$ & 17E3 & $\begin{array}{l}\text { gwm108- } \\
\text { wmc787 }\end{array}$ & & & 10.43 & & & -0.09 \\
\hline & $\begin{array}{l}\text { Qgw- } \\
4 D .1\end{array}$ & $15 \mathrm{E} 1$ & $\begin{array}{l}\text { barc334- } \\
\text { wmc285 }\end{array}$ & 24.28 & & & 0.18 & & \\
\hline & $\begin{array}{l}\text { Qgw- } \\
5 D .1\end{array}$ & $15 \mathrm{E} 1$ & $\begin{array}{l}\text { cfd67- } \\
\text { cfd40 }\end{array}$ & 16.56 & & & 0.16 & & \\
\hline & $\begin{array}{l}\text { Qgw- } \\
5 D .2\end{array}$ & $15 \mathrm{E} 1$ & $\begin{array}{l}\text { gwm174- } \\
\text { gwm583 }\end{array}$ & 14.39 & & & 0.16 & & \\
\hline & $\begin{array}{l}\text { Qgw- } \\
6 D .1\end{array}$ & $15 \mathrm{E} 1$ & $\begin{array}{l}\text { barc196- } \\
\text { barc54 }\end{array}$ & 16.58 & & & 0.17 & & \\
\hline & $\begin{array}{l}\text { Qgw- } \\
6 D .2\end{array}$ & 15E1, 16E1 & $\begin{array}{l}\text { barc54- } \\
\text { cfd42 }\end{array}$ & 9.03 & 9.92 & & 0.11 & 0.16 & \\
\hline \multirow[t]{8}{*}{ TGW } & $\begin{array}{l}\text { Qtgw- } \\
-2 A\end{array}$ & 17E1 & $\begin{array}{l}\text { gwm382- } \\
\text { gwm526 }\end{array}$ & & & 32.56 & & & -5.61 \\
\hline & $\begin{array}{l}\text { Qtgw- } \\
2 B\end{array}$ & $16 \mathrm{E} 1$ & $\begin{array}{l}\text { wmc441- } \\
\text { wmc317 }\end{array}$ & & 17.14 & & & -3.35 & \\
\hline & $\begin{array}{l}\text { Qtgw- } \\
3 B .3\end{array}$ & $16 \mathrm{E} 1$ & $\begin{array}{l}\text { wmc787- } \\
\text { wmc687 }\end{array}$ & & 11.35 & & -2.57 & -2.57 & \\
\hline & $\begin{array}{l}\text { Qtgw- } \\
4 B\end{array}$ & $15 \mathrm{E} 1$ & $\begin{array}{l}\text { gpw7026- } \\
\text { barc20 }\end{array}$ & 10.27 & & & 1.15 & & \\
\hline & $\begin{array}{l}\text { Qtgw- } \\
5 D .1\end{array}$ & $15 \mathrm{E} 1$ & $\begin{array}{l}\text { wmc357- } \\
\text { wmc97 }\end{array}$ & 27.53 & & & 1.87 & & \\
\hline & $\begin{array}{l}\text { Qtgw- } \\
5 D .2\end{array}$ & 15E1, 17E1 & $\begin{array}{l}\text { cfd266- } \\
\text { cfd67 }\end{array}$ & 10.48 & & 12.27 & 1.60 & & 1.65 \\
\hline & $\begin{array}{l}\text { Qtgw- } \\
6 D .1\end{array}$ & $15 \mathrm{E} 1$ & $\begin{array}{l}\text { barc196- } \\
\text { barc54 }\end{array}$ & 9.49 & & & 1.56 & & \\
\hline & $\begin{array}{l}\text { Qtgw- } \\
6 D .2\end{array}$ & $16 \mathrm{E} 1$ & $\begin{array}{l}\text { gdm132- } \\
\text { cfd49 }\end{array}$ & & 14.98 & & & 2.31 & \\
\hline
\end{tabular}

Note: CC, chlorophyll content; LWC, leaf water content; GW, grain weight per spike; TGW, thousand-grain weight. E1, Yinchuan; E2, Hohhot; E3, Urumqi.

Table 4 QTL locating of related physiological and agronomical traits under combined drought and heat stresses in multiple locations in three different years. 


\begin{tabular}{|c|c|c|c|c|c|c|c|c|c|}
\hline \multirow[t]{2}{*}{ Traits } & \multirow[t]{2}{*}{ QTL } & \multirow[t]{2}{*}{ Environments } & \multirow[t]{2}{*}{ Marker interval } & \multicolumn{2}{|c|}{ Variation (\%). } & \multicolumn{4}{|c|}{$\underline{\text { Additive effect }}$} \\
\hline & & & & 2015 & 2016 & 2017 & 2015 & 2016 & 2017 \\
\hline \multirow[t]{10}{*}{$\mathrm{CC}$} & $Q c c-2 A .1$ & 15E1, 16E1 & gwm382-gwm526 & 18.42 & 33.01 & & 1.74 & 7.09 & \\
\hline & Qcc-2A.2 & 15E1, 16E1, 17E3 & gwm294-wmc644 & 10.12 & 12.18 & 26.14 & 0.96 & 3.23 & 5.56 \\
\hline & $Q c c-3 A$ & 16E1, 17E1 & gpw5042-gpw2169 & & 15.19 & 15.20 & & 2.19 & 1.69 \\
\hline & $Q c c-4 A$ & 16E1, 17E1 & wmc262-barc343 & & 18.42 & 10.54 & & -3.81 & -3.97 \\
\hline & $Q c c-2 B$ & 15E1 & wmc441-wmc317 & 22.13 & & & -2.23 & & \\
\hline & $Q c c-2 B .1$ & 15E1 & barc167-gwm388 & 11.57 & & & 2.26 & & \\
\hline & Qcc-3B.2 & 16E1 & wmc1-wmc307 & & 14.17 & & & -2.09 & \\
\hline & $Q c c-4 B .2$ & $16 \mathrm{E} 2$ & gpw7556-wmc349 & & 34.35 & & & 6.31 & \\
\hline & Qcc-3D.1 & 16E1 & gpw4451-barc323 & & 9.09 & & & -2.89 & \\
\hline & $Q c c-4 D$ & 15E1, 16E1 & wmc285-gpw7795 & 16.14 & 24.15 & & 3.41 & 4.95 & \\
\hline \multirow[t]{13}{*}{ LWC } & QIwc-2A.2 & $16 \mathrm{E} 2$ & Gwm294-wmc644 & & 21.99 & & & 5.67 & \\
\hline & QIwc-2A.3 & $16 \mathrm{E} 2$ & wmc522-gwm359 & & 13.79 & & & 4.47 & \\
\hline & Q/Wc-3A & $16 \mathrm{E} 3$ & wmc264-wmc559 & & 30.65 & & & 13.13 & \\
\hline & Q/wc-2B.1 & $16 \mathrm{E} 3$ & wmc661-wmc441 & & 31.99 & & & -10.87 & \\
\hline & Q/wc-2B.2 & $16 \mathrm{E} 1$ & cfa2278-barc167 & & 13.91 & & & 1.29 & \\
\hline & Q/wc-3B.3 & 15E1 & barc77-gwm340 & 22.14 & & & 0.86 & & \\
\hline & QIwc-3B.4 & $16 \mathrm{E} 1$ & wmc787-wmc687 & & 20.86 & & & 0.85 & \\
\hline & Q/wC-4B & 16E1 & barc174-wmc657 & & 15.35 & & & 1.81 & \\
\hline & QIwc-7B.1 & 15E1, 16E2, 17E1 & wmc83-wmc276 & 30.81 & 38.51 & 30.67 & 3.69 & 10.58 & 14.19 \\
\hline & Q/wc-7B.2 & $16 \mathrm{E} 3$ & cfa2106-gwm333 & & 32.95 & & & -12.27 & \\
\hline & Q/wc-7B.3 & 15E1 & barc140-gwm297 & 30.01 & & & 2.11 & & \\
\hline & Q/wC-2D & 17E3 & wmc111-wmc25 & & & 10.32 & & & 3.50 \\
\hline & QIwc-4D.1 & $16 \mathrm{E} 2$ & wmc720-wmc331 & & 11.78 & & & 0.97 & \\
\hline \multirow[t]{11}{*}{ GW } & $Q g w-2 A .2$ & 15E1 & gwm294-wmc644 & 19.40 & & & 0.16 & & \\
\hline & $Q g w-2 A .3$ & 15E1, 17E2 & gwm425-wmc522 & 21.75 & & 19.78 & 0.21 & & 0.75 \\
\hline & $Q g w-3 A$ & $16 \mathrm{E} 3$ & wmc664-wmc264 & & 25.43 & & & -0.25 & \\
\hline & $Q g w-3 B .1$ & 15E1, 16E2, 17E1 & wmc808-wmc78 & 11.16 & 29.33 & 13.53 & -0.17 & -0.36 & -0.21 \\
\hline & Qgw-3B.3 & 15E1 & barc77-gwm340 & 35.67 & & & -0.23 & & \\
\hline & $Q g w-4 B .1$ & 15E1 & gwm251-wmc652 & 10.99 & & & 0.15 & & \\
\hline & $Q g w-4 B .2$ & 15E1, 17E3 & barc174-wmc657 & 13.84 & & 15.67 & -0.25 & & -0.14 \\
\hline & Qgw-4B.3 & $16 \mathrm{E} 3$ & barc20-gwm495 & & 16.34 & & & 0.16 & \\
\hline & Qgw-7B.1 & $15 \mathrm{E} 1$ & barc176-gwm400 & 11.02 & & & -0.15 & & \\
\hline & $Q g w-4 D$ & $15 \mathrm{E} 1$ & wmc720-wmc331 & 9.78 & & & -1.34 & & \\
\hline & $Q g w-6 D .2$ & 15E1, 16E1 & barc54-cfd42 & 11.18 & 9.39 & & 0.15 & 0.15 & \\
\hline
\end{tabular}

Page $18 / 23$ 


\begin{tabular}{|c|c|c|c|c|c|c|c|c|c|}
\hline \multirow[t]{11}{*}{ TGW } & Qtgw-2A.2 & 17E3 & gwm294-wmc644 & & & 10.05 & & & 1.39 \\
\hline & $Q \operatorname{tg} w-2 B$ & 15E1, 16E1, 17E1 & wmc441-wmc317 & 22.13 & 13.27 & 21.90 & -2.22 & -2.75 & -2.72 \\
\hline & Qtgw-3B.2 & $16 \mathrm{E} 2$ & wmc787-wmc687 & & 14.25 & & & -1.91 & \\
\hline & Qtgw-7B & 15E1, 16E1 & wmc83-wmc276 & 27.21 & 33.01 & & 5.83 & -10.11 & \\
\hline & Qtgw-2D & $16 \mathrm{E} 3$ & wmc111-wmc25 & & 9.55 & & & 2.31 & \\
\hline & Qtgw-3D & 15E1, 16E3 & barc323-wmc552 & 13.18 & 8.81 & & -1.85 & -1.87 & \\
\hline & Qtgw-3D.2 & $15 \mathrm{E} 1$ & gpw4451-barc323 & 13.18 & & & -1.85 & & \\
\hline & Qtgw-4D & $15 \mathrm{E} 1$ & wmc720-wmc331 & 9.78 & & & -1.34 & & \\
\hline & Qtgw-6D.2 & $15 \mathrm{E} 1$ & gdm132-cfd49 & 13.74 & & & 1.72 & & \\
\hline & Qtgw-6D.3 & $15 \mathrm{E} 1$ & barc175-barc204 & 9.38 & & & 1.56 & & \\
\hline & Qtgw-6D.4 & $15 \mathrm{E} 1$ & gdm132-cfd49 & 13.74 & & & 1.70 & & \\
\hline
\end{tabular}

Note: CC, chlorophyll content; LWC, leaf water content; GW, grain weight per spike; TGW, thousand-grain weight. E1, Yinchuan; E2, Hohhot; E3, Urumqi.

Table 5 QTL localization of related physiological and agronomic traits under normal conditions in multiple locations in three different years. 


\begin{tabular}{|c|c|c|c|c|c|c|c|c|c|}
\hline \multirow[t]{2}{*}{ Traits } & \multirow{2}{*}{ QTL } & \multirow[t]{2}{*}{ Environments } & \multirow[t]{2}{*}{ Marker interval } & \multicolumn{2}{|c|}{ Variation (\%). } & \multicolumn{4}{|c|}{ Additive effect } \\
\hline & & & & 2015 & 2016 & 2017 & 2015 & 2016 & 2017 \\
\hline \multirow[t]{4}{*}{$\mathrm{CC}$} & $Q c c-2 A .1$ & 15E1 & gwm382-gwm526 & 17.61 & & & 1.39 & & \\
\hline & $Q c c-2 A .2$ & 15E1, 16 E2, 17E3 & gwm294-wmc644 & 29.01 & 26.29 & 20.51 & 1.65 & 1.91 & 3.24 \\
\hline & $Q c c-3 B .1$ & $16 \mathrm{E} 2$ & wmc808-wmc78 & & 28.32 & & & -1.70 & \\
\hline & Qcc-7B.1 & 16E2, 17E2 & wmc83-wmc276 & & 33.86 & 29.29 & & 8.81 & 10.91 \\
\hline \multirow[t]{7}{*}{ LWC } & Q/wC-2A.1 & 15E1 & gwm382-gwm526 & 20.62 & & & 2.60 & & \\
\hline & QIWC-2A.2 & 15E2 & gwm294-wmc644 & 11.71 & & & 4.24 & & \\
\hline & QIwc-2A.3 & $16 \mathrm{E} 3$ & barc309-gwm122 & & 11.34 & & & -6.25 & \\
\hline & QIwc-3B.2 & $16 \mathrm{E} 2$ & wmc787-wmc687 & & 19.59 & & & 1.36 & \\
\hline & Q/WC-4D & 15E1 & wmc720-wmc331 & 14.97 & & & 1.58 & & \\
\hline & Q/Wc-7B.1 & 15E1, 16E1, 17E1 & wmc83-wmc276 & 28.89 & 34.72 & 29.97 & -3.55 & 5.62 & 8.42 \\
\hline & Q/Wc-7B & $15 \mathrm{E} 1$ & barc140-gwm297 & 11.65 & & & -1.45 & & \\
\hline \multirow[t]{5}{*}{ GW } & Qgw-2A.2 & 15E1, 16E2, 17E3 & gwm294-wmc644 & 21.96 & 11.83 & 15.31 & 0.21 & 0.15 & 0.89 \\
\hline & $Q g w-3 B .1$ & $16 \mathrm{E} 2$ & wmc808-wmc78 & & 18.17 & & & -0.30 & \\
\hline & $Q g w-3 B .2$ & $16 \mathrm{E} 2$ & wmc787-wmc687 & & 11.34 & & & -0.22 & \\
\hline & Qgw-7B.1 & 17E3 & wmc83-wmc276 & & & 18.74 & & & -0.39 \\
\hline & $Q g w-7 B$ & 17E3 & barc140-gwm297 & & & 28.92 & & & -0.19 \\
\hline \multirow[t]{4}{*}{ TGW } & Qtgw-2A.3 & 16E1 & barc309-gwm122 & & 9.75 & & & -1.936 & \\
\hline & Qtgw-3B.1 & $16 \mathrm{E} 2$ & wmc808-wmc78 & & 29.16 & & & -3.33 & \\
\hline & Qtgw-3B.2 & 15E1, 16E2, 17E3 & wmc787-wmc687 & 8.23 & 11.34 & 8.31 & -1.34 & -0.22 & -1.80 \\
\hline & Qtgw-6D & $15 \mathrm{E} 1$ & barc196-barc54 & 10.49 & & & 1.56 & & \\
\hline
\end{tabular}

Note: CC, chlorophyll content; LWC, leaf water content; GW, grain weight per spike; TGW, thousand-grain weight. E1, Yinchuan; E2, Hohhot; E3, Urumqi.

\section{Figures}



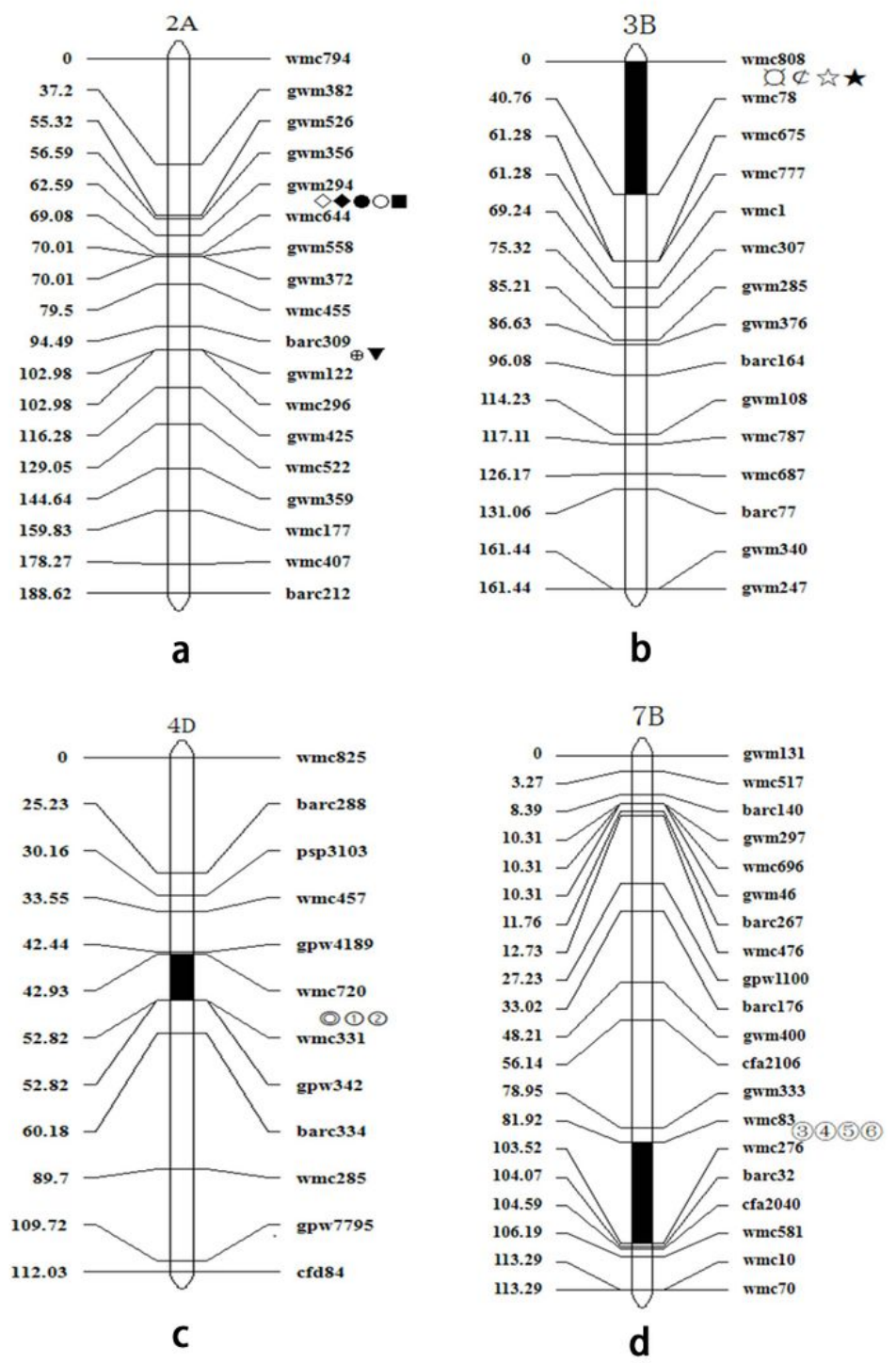

\section{Figure 1}

Chromosomal location of genetic overlapping QTLs associated with drought tolerance of spring wheat during three different years. a. Location of genetic overlapping QTLs on chromosome 2A. 『: 15E2 drought stress for CC; $₫:$ 16E1 drought stress for CC; $\bullet$ : 15E1 drought stress for GW; $\bullet$ : 16E2 drought stress for GW; $\mathbf{\square}$ : 17E3 drought stress for GW; $\oplus$ : 16E2 drought stress for LWC; $\mathbf{\nabla}$ : 16E1 drought stress for TGW. b. Location of genetic overlapping QTLs on chromosome 3B. d: 16E2 drought stress for LWC; 『: 15E1 drought stress for GW; 『: 16E2 drought stress for GW; 『: 17E3 drought stress for GW. c. Location of genetic overlapping QTLs on chromosome 4D. 『: 16E1 drought stress for LWC; 『: 17E2 drought stress for LWC; 『: $15 E 1$ drought stress for GW. d. Location of genetic overlapping QTLs on chromosome 7B. 『: 15E2 drought stress for LWC; 『: 16E3 drought stress for LWC; 『: 17E1 drought

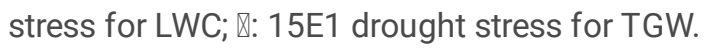



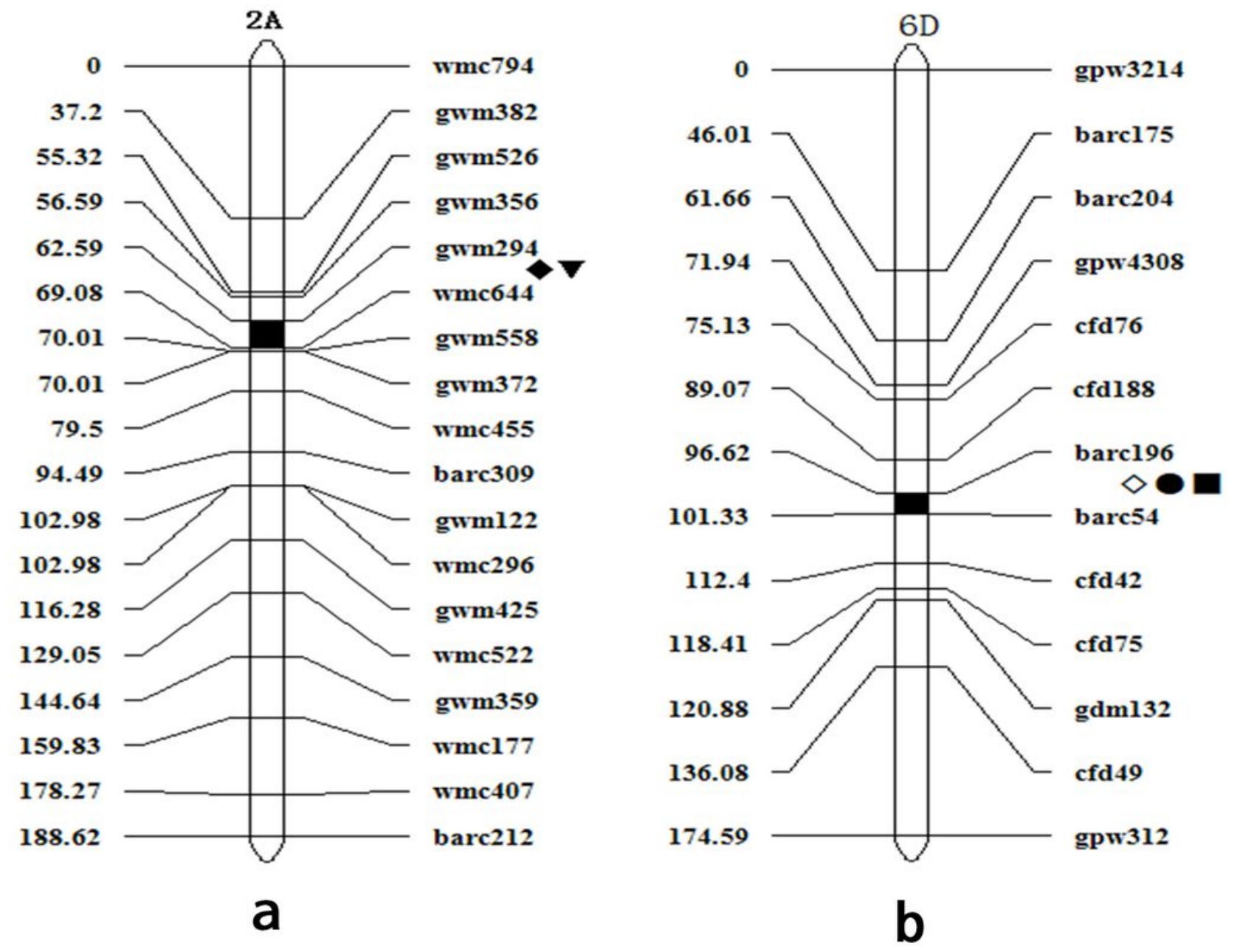

Figure 2

Chromosomal location of genetic overlapping QTLs associated with heat tolerance of spring wheat during three different years. a. Location of genetic overlapping QTLs on chromosome 2A. 『: 15E1 heat stress for CC; $\mathbf{\nabla}$ : $15 \mathrm{E} 1$ heat stress for TGW. b. Location of

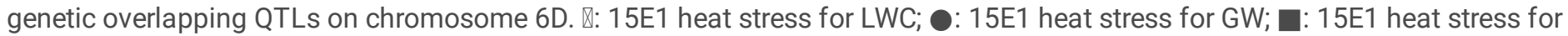
TGW. 


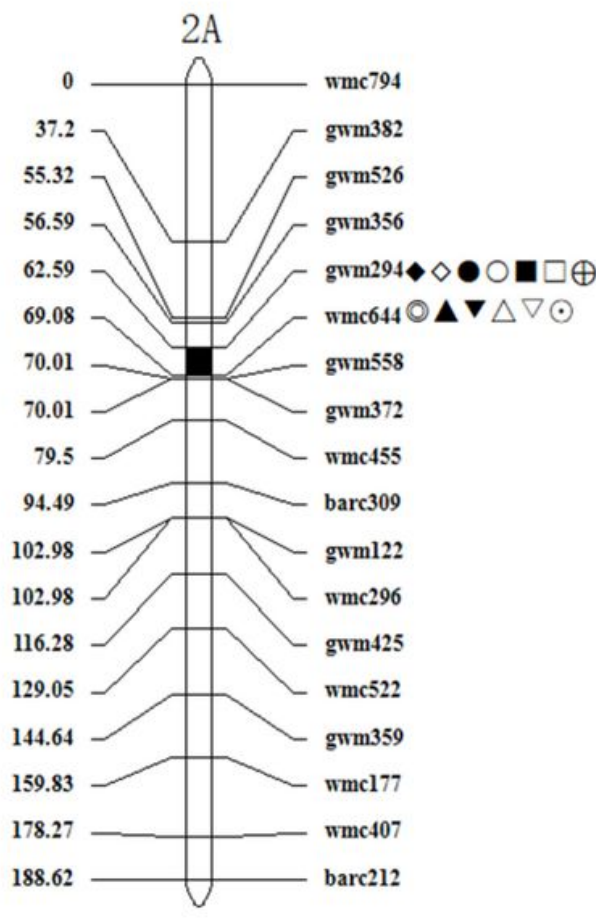

a

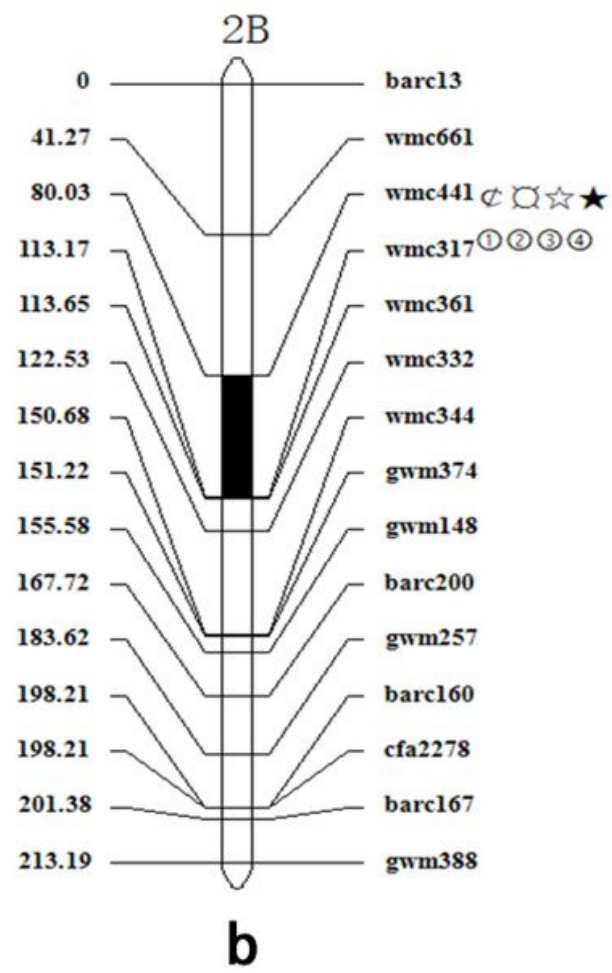

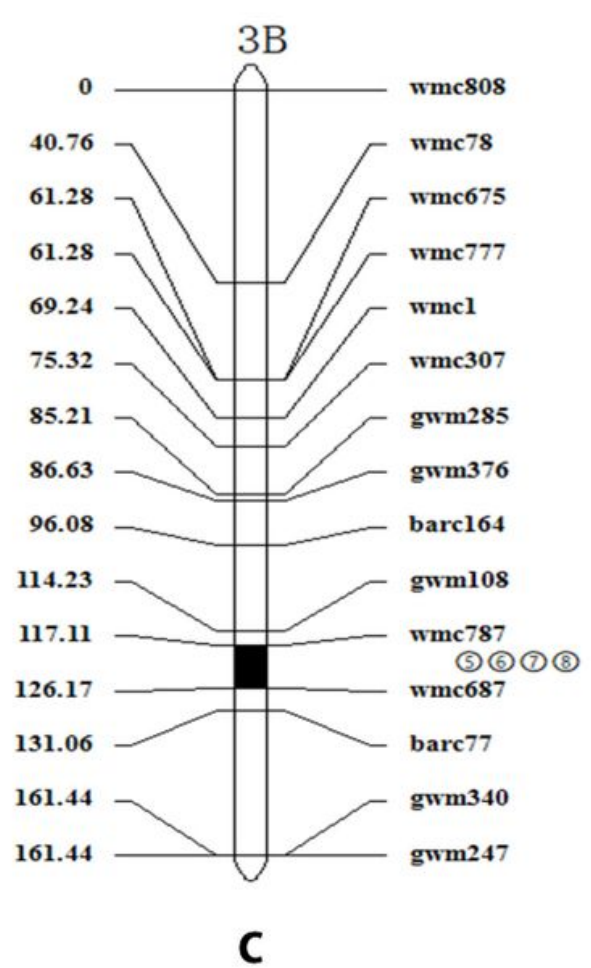

\section{Figure 3}

Chromosomal location of genetic overlapping QTLs associated with combined drought and heat tolerance of spring wheat during three different years. a. Location of genetic overlapping QTLs on chromosome 2A. 『: 15E2 drought stress for CC; 『: 16E1 drought stress for CC; $\bullet: 15 E 1$ drought stress for GW; $\bullet$ : 16E2 drought stress for GW; $\mathbf{\square}$ : 17E3 drought stress for GW; $\square$ : $15 E 1$ heat stress

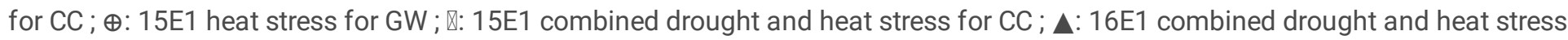
for $C C ; \nabla: 17 E 3$ combined drought and heat stress for CC; $\triangle:$ 16E2 combined drought and heat stress for LWC; $\nabla: 15 E 1$ combined drought and heat stress for GW; $\odot: 17 E 3$ combined drought and heat stress for TGW. b. Location of genetic overlapping QTLs on chromosome 2B. 邓: 15E1 drought stress for TGW; a: 16E1 drought stress for TGW; 『: 16E1 heat stress for CC; 邓: 16E1 heat stress for CC; $邓: 15 E 1$ combined drought and heat stress for CC; $:$ : 15E1combined drought and heat stress for TGW; $邓: 16 E 1$ combined drought and heat stress for TWG; $邓:$ 17E1 combined drought and heat stress for TWG. c. Location of genetic overlapping QTLs on chromosome 3B.囚: 17E3 drought stress for TGW; 『: 16E2 heat stress for LWC; 『: $16 E 1$ heat stress for TGW; 『: 15E1 combined drought and heat stress for LWC. 